\title{
Towards understanding and managing chronic cough
}

\author{
Authors: Imran Satia, ${ }^{A}$ Huda Badri, ${ }^{A}$ Bashar Al-Sheklly, ${ }^{A}$ Jaclyn Ann Smith ${ }^{B}$ and Ashley A Woodcock ${ }^{B}$
}

Chronic cough is a common and troublesome condition affecting approximately $12 \%$ of the general population. It is associated with poor quality of life with psychological, social and physical consequences. Patients typically complain of a dry irritating cough, driven by a strong urge to cough associated with a sensation or irritation located in the throat. Treatment of potential 'causes', ie asthma, gastrooesophageal reflux disease and rhino-sinusitis, may produce a complete or partial response, but the response of some patients to opiates and alpha-2-delta ligand antagonists (gabapentin and pregabalin) supports the concept that this is primarily a neurological disorder, characterised by hyperresponsiveness of the nerves. Novel and highly effective neuronal treatments are in development and offer hope of better symptom control with fewer side effects within a few years. This review focuses on understanding the mechanism of chronic cough, current management approaches and research that may lead to novel therapies.

KEYWORDS: Capsaicin, chronic cough, cough hypersensitivity syndrome, vagus nerve, $\mathrm{P} 2 \times 3$

\section{Introduction}

Cough is the single most common reason for seeking medical attention and the lack of suitable anti-tussives makes this a major unmet clinical need. Many patients experience acute coughing lasting up to 3 weeks after a viral respiratory tract infection. However, a substantial proportion of the population (12-16\%) suffer from chronic cough (lasting more than 8 weeks) with detrimental effects on quality of life..$^{1-3}$ The stereotypical patient is a woman in her 50s with a nonproductive cough for many years. Chronic cough is twice as common in women as in men. ${ }^{4}$ Chronic cough often occurs in distressing bouts, which the patient cannot control, and is usually preceded by an irresistible urge to cough associated with

Authors: ${ }^{\text {A }}$ clinical research fellow, Division of Infection, Immunity and Respiratory Medicine, University of Manchester, and Manchester Academic Health Sciences Centre. University Hospital of South Manchester NHS Foundation Trust, Manchester, UK; ${ }^{\text {B }}$ professor and consultant in respiratory medicine, Division of Infection, Immunity and Respiratory Medicine, University of Manchester, and Manchester Academic Health Sciences Centre. University Hospital of South Manchester NHS Foundation Trust, Manchester, UK an irritating sensation in the throat. Cough can be precipitated by changes in temperature, strong smells from perfumes or aerosols, dust and passive smoking. ${ }^{5,6}$ Chronic cough has major psychological, social and physical consequences. Patients are embarrassed socially and in the work place. They are physically exhausted by the frequent prolonged coughing bouts, and women can have distressing stress incontinence. The treatment options have been limited. In the USA, over $\$ 7$ billion is spent each year on over-the-counter cough and cold medications, most of which contain dextromethorphan, which has very limited if any clinical effectiveness. ${ }^{7}$ Understanding the mechanisms underpinning coughing in order to develop novel treatments is a significant challenge to the medical profession and pharmaceutical industry.

\section{Neurophysiology of cough}

Cough is normally a defensive reflex that protects the airways. It is a response to an inhaled foreign body or excessive mucus, and to noxious and harmful environmental irritants. Activated sensory airway nerves transmit information via the vagus nerve to first synapse in the brainstem, which rapidly initiates the motor cough response.

The cough reflex is thought to involve two main subtypes of sensory vagal afferent nerves. ${ }^{8}$ The first subtype is c-fibres; these form networks of unmyelinated nerves throughout the airways and are characteristically sensitive to capsaicin (chilli pepper extract) through activation of the transient receptor potential vanilloid type 1 (TRPV1) receptor and other irritant chemicals. They can also respond to other stimuli such as heat, acidity and inflammatory mediators. The second type, myelinated sub-epithelial A $\delta$ fibres, are found in the proximal airways and respond to mechanical stimuli, osmolarity and acidity but do not typically express TRPV1, and are normally insensitive to capsaicin and inflammatory mediators. The morphology of these airway nerves has recently been delineated in human airway tissue and shows remarkable similarity to that seen in animal models (Fig 1). ${ }^{9}$

Stimulating these airway nerves generates action potentials that synapse in the nucleus tractus solitarius (NTS) and paratrigeminal nucleus of the brainstem. ${ }^{10}$ These afferent nerves then activate complex neural networks, projecting to cortical and sub-cortical areas responsible for sensations of airway irritation and the urge to cough and ultimately, if the stimulus is sufficient, results in coughing via activation of spinal motor nerves to the diaphragm, intercostal muscles and larynx (Fig 2). Importantly, coughing can also 

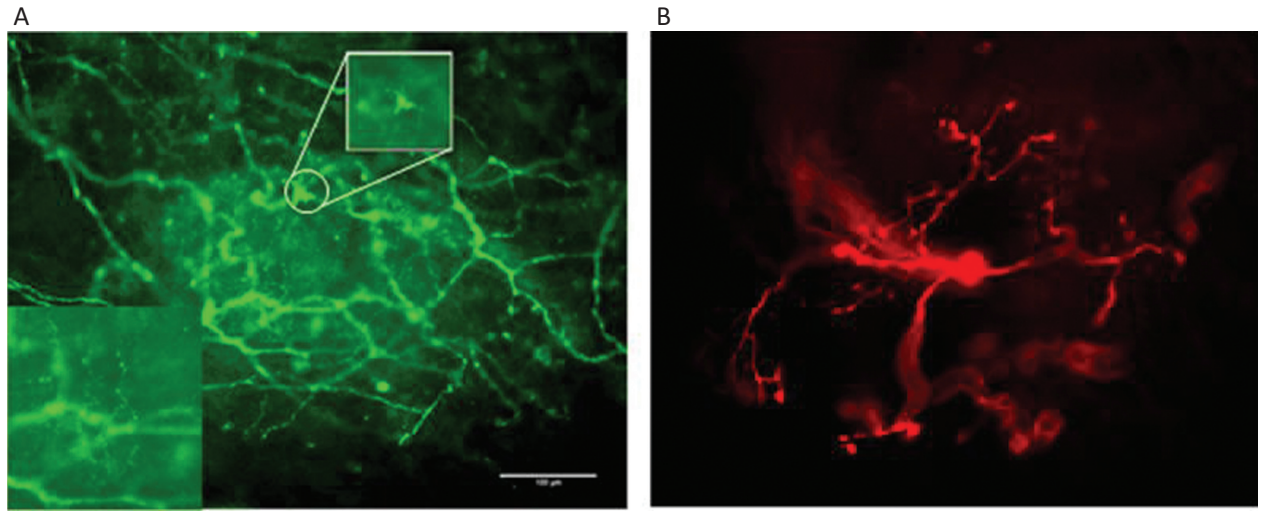

Fig 1. Structure of human airway nerves. A - thin unmyelinated $\mathrm{c}$-fibres located near the epithelial membrane; B - sub-epithelial myelinated A $\delta$ fibres. Reprinted with permission of the American Thoracic Society. ${ }^{9}$ Copyright (c) 2016 American Thoracic Society.

be initiated voluntarily without any peripheral stimulus or precipitating sensations, and in some cases voluntarily supressed. ${ }^{11}$ Thus, the potential drivers of excessive cough could originate either in the peripheral nerves or central nervous system, including the brainstem. There have been case report associations noted between chronic cough and conditions affecting the autonomic nervous system such as Holmes-Adie syndrome and autosomal dominant hereditary sensory neuropathy. ${ }^{12-15}$

\section{Hypersensitive or hyper-responsive cough?}

A recent consensus statement has suggested the term 'cough hypersensitivity syndrome' be used to describe patients with chronic cough. ${ }^{16}$ However, evidence from experimentally evoked cough suggests that the neuronal pathways exhibit hyper-responsiveness rather than hypersensitivity. ${ }^{17}$ In pharmacokinetic terms, the dose response curve is

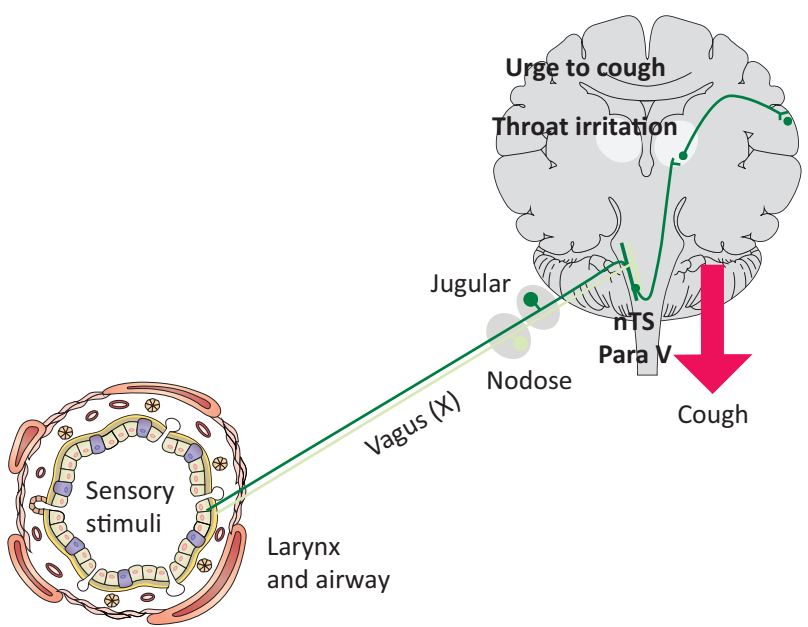

Fig 2. Schematic diagram representing the cough reflex. Vagal afferents transmit stimuli from the airways to the nucleus tractus solitarius (nTS) and paratrigeminal nucleus (Para V) in the brainstem. Neuronal signals are then transmitted to the somatosensory cortex via the thalamus causing throat irritation and urge to cough. These sensations, if great enough, lead to cough via activation of spinal motor neurons. predominantly shifted upwards with much less left-shift, ie the dose required to evoke a cough is marginally reduced, but the maximal number of coughs to a given stimulus is substantially increased (Fig 3). This is reflected in what patients describe; anecdotally, patients complain of an inability to stop coughing and quality of life is most severely impacted by the length and severity of coughing bouts.

\section{Aetiology of chronic cough}

Chronic cough can occur in many common respiratory conditions such as asthma, bronchitis in smokers and chronic obstructive pulmonary disease (COPD), where the cough is typically related to the pathophysiology of the disease, eg excessive airway mucus and inhalation of irritants in cigarette smoke for COPD. ${ }^{18}$ Other causes include eosinophilic

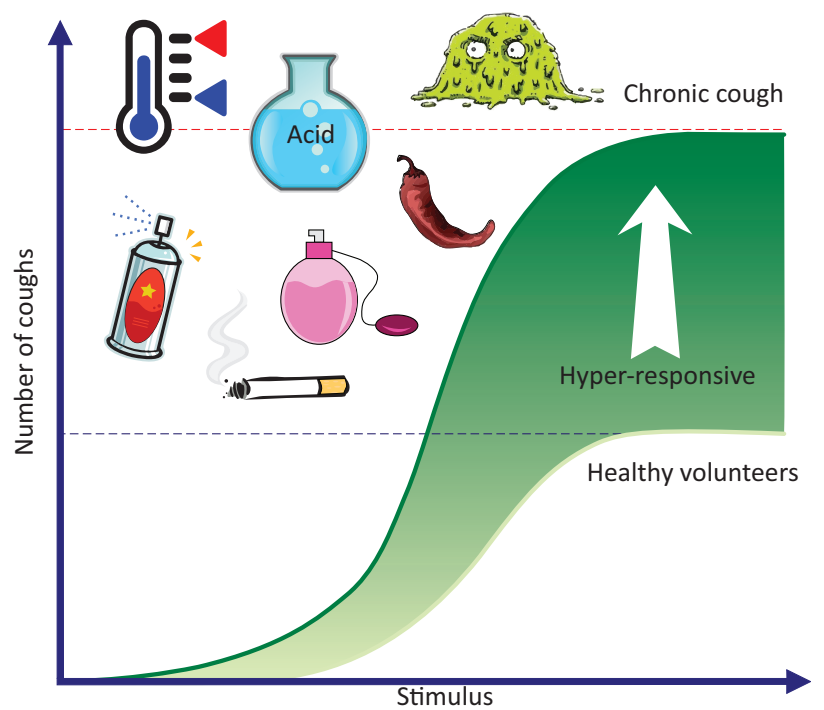

Fig 3. Schematic diagram representing cough hyper-responsiveness to airway stimuli displayed by chronic cough patients. Compared with healthy volunteers, patients with chronic cough predominantly exhibit heightened cough responses to airway stimuli, such as inhaled capsaicin and citric acid, in cough challenge test and report coughing triggered by changes in temperature, perfumes, aerosols, smoke and to small volumes of mucus. 
bronchitis, interstitial lung diseases, bronchiectasis or use of angiotensin-converting enzyme inhibitors. Extra-pulmonary diseases, such as gastro-oesophageal reflux disease (GORD) and post-nasal drip secondary to rhinosinusitis, can also be identified as potential triggers.

Observational studies detailing the aetiology of chronic cough in patients presenting to specialist cough clinics show large variations in the prevalence of asthma (6-36\%), GORD $(0-41 \%)$ and rhinitis $(8-56 \%) .{ }^{19}$ Furthermore, up to a quarter of patients may have multiple aetiologies combined. ${ }^{20}$

In the past three decades, this diagnostic triad of asthma, GORD or rhinosinusitis have been asserted to be the 'cause' of chronic cough. However, the vast majority of patients with these common conditions do not complain of coughing or have features suggestive of cough hypersensitivity. Treatment of these conditions in patients with chronic cough may improve cough but rarely stops it completely. After numerous investigations, some patients have no evidence of any of the above causes, and hence the use of the term idiopathic chronic cough. $^{21}$

Given our recent understanding of the neurophysiology of cough, it seems likely that neuronal dysfunction is the primary cause of chronic cough. Indeed, evidence for such has been demonstrated by heightened cough responses to inhaled capsaicin in patients with chronic cough ${ }^{17,22}$ and asthma. ${ }^{23}$ In the context of cough hyper-responsiveness, cough can be triggered by endogenous factors (asthma, GORD, post-nasal drip, even speaking and laughing) or exogenous factors (eg cold air, passive smoking, deodorants etc).

\section{Management approach to chronic cough}

There are many international guidelines based on consensus opinion and observational data that describe the approaches taken in managing patients presenting with chronic cough. ${ }^{19,24-26}$ The applicability of these guidelines will vary based on the availability of specialised investigations and clinical experience at primary, secondary and tertiary levels. Despite the complexity of these diagnostic algorithms, the basic approach can be summarised in four main steps, ${ }^{27}$ which can be performed with appropriate referral made to secondary or tertiary care if needed.

\section{Exclude and treat obvious causes}

A detailed history and examination should aim to identify obvious causes and exclude sinister pathologies, such as malignancy. The duration and nature of symptoms is useful, as a cough lasting less than 3 weeks is usually secondary to an upper respiratory tract viral infection, while a cough lasting 3-8 weeks can often be associated with a prolonged post-infectious period, and is occasionally secondary to Mycoplasma or Bordetella pertussis. The majority of acute coughs are self-limiting and unfortunately very few clinical studies exist to support the use of over the counter (OTC) anti-tussives, which generally contain dextromethorphan. ${ }^{7}$ Smoking, even in otherwise healthy subjects, has now been shown to be associated with an increased frequency of objective 24-hour coughs. ${ }^{18}$ Approximately $15 \%$ of patients on an angiotensin-converting enzyme inhibitor may develop a chronic cough and hence changing to the alternative angiotensin receptor blocker would be appropriate, ${ }^{28}$ but the improvement may take several months to take effect. For patients with chronic cough, an initial evaluation should also include a chest radiograph and spirometry. It is important to differentiate a dry, minimally productive cough and a cough with a high daily volume of mucus. The latter would suggest bronchiectasis and would need further investigations to confirm.

\section{Investigate and treat common triggers of chronic cough}

If the chest radiograph, spirometry and clinical examination are normal, then investigations for asthma, GORD and rhinosinusitis should be considered. Although most patients with asthma have a history of wheeze and shortness of breath as the predominant feature, some patients have cough as the key feature and have been previously coined 'cough-variant asthma. ${ }^{29,30}$

Objective evidence of asthma requires evidence of variable airflow obstruction - such as peak flow variability of more than $20 \%$ or reversibility to salbutamol of more than $12 \%$. However, both of these investigations have a very low negative predictive value and, therefore, further investigation of bronchial hyper-responsiveness (BHR) may be required using either methacholine or histamine inhalational challenge. These tests are not always available in all secondary care settings so a treatment trial of inhaled corticosteroids for 6 weeks is often a more pragmatic approach. In our experience, a trial of inhaler therapy can be limited by the patient's technique and compliance given inhaled treatments often trigger coughing; an alternative is to prescribe 14 days of oral steroids, prednisolone $30 \mathrm{mg}$ daily is our preference. However, a dramatic improvement in cough still leaves some diagnostic uncertainty; a response to inhaled therapy suggests either asthma or eosinophilic bronchitis (EB) and a response to oral steroids could also indicates allergic nasal disease or interstitial lung diseases.

Elevated eosinophils ( $>3 \%)$ in the airways in the absence of BHR suggests EB, which has been reported in up to $13 \%$ of patients attending cough clinics. ${ }^{31}$ This can be evaluated in samples of induced sputum or from broncho-alveolar lavage (BAL) performed at bronchoscopy, but performing different cell counts on these airway samples is not routinely available in many hospitals. An easier alternative, if available, is exhaled nitric oxide in breath as a surrogate marker to assess airway eosinophils, but its utility in aiding diagnosis in patients with chronic cough has not yet been systematically evaluated. Overall, the key recommendation is that inhaled steroids be trialled in patients who have evidence of BHR or airway eosinophilia in sputum, BAL or exhaled nitric oxide.

Objectively investigating GORD is a challenge and previous guidelines advised empirical acid suppression treatment. A 2011 Cochrane review concluded 'in adults, there is insufficient evidence to conclude definitely that GERD treatment with PPI is universally beneficial for cough associated with GORD'. ${ }^{32}$ However, those that demonstrate objective evidence of increased oesophageal acid exposure on $\mathrm{pH}$ monitoring or complained of heartburn were most likely to benefit from proton pump inhibitor (PPI) therapy in a retrospective review of the PPI studies. ${ }^{33,34}$ In our practice, 
we use a trial of twice daily PPI 30 minutes before food, and ranitidine $300 \mathrm{mg}$ at bedtime for 8 weeks.

The mechanisms linking cough and reflux are still unclear. No clear evidence exists to support the concept of microaspiration of refluxate into the airways nor that refluxate reaches the proximal oesophagus or enters the larynx/ pharynx. ${ }^{35,36}$ Furthermore, most chronic cough patients have similar numbers of reflux events to healthy controls; however, in $50 \%$ there is a temporal relationship between reflux and cough, ${ }^{37}$ suggesting that in some patients stimulation of oesophageal afferents may be capable of triggering coughing, again consistent with sensitisation of neuronal pathways. No evidence exists for the use of pro-kinetics such as domperidone, which has recently been associated with ventricular arrhythmias and sudden cardiac death. Likewise, metoclopramide should be avoided because of its neurological side effects and the lack of clear indication for use in chronic cough.

Patients with chronic cough also often report sensations of post-nasal drip so guidelines recommend nasal corticosteroids and anti-histamines for those thought to have underlying allergic rhinitis and antibiotics/decongestants for sinusitis. ${ }^{24}$ Many patients with chronic cough are often assessed by ear, nose and throat surgeons and, upon inspection, the larynx are found to be red and inflamed; this is often attributed to laryngo-pharyngeal reflux (LPR) secondary to gastric refluxate reaching the larynx. However as discussed earlier, evidence of gastric reflux reaching the larynx is lacking and patients who are often coughing hundreds of times may show signs of trauma to the larynx.

\section{Exclude and treat rarer triggers of chronic cough}

For those without a clear diagnosis or chronic cough that is refractory to treatment of associated conditions, it is important to exclude rarer conditions by performing a high-resolution computerised tomography scan of the chest. This can reveal interstitial lung diseases not easily visible on chest radiograph, eg pulmonary fibrosis, sarcoidosis and bronchiectasis. When other investigations are normal, bronchoscopy is performed in all our patients to assess vocal cord movements, identify tracheobronchomalacia or tracheopathia osteochondroplastica and take BAL and biopsies to evaluate eosinophils. Thick purulent secretions are occasionally found in patients with a productive cough; these secretions are cleared and cultured for bacteria, fungus and acid-fast bacilli. Bronchoscopy can also reveal naso-pharyngeal pathologies such rhinitis, polyps and large tonsils, which the patient may benefit from having removed. $^{38}$

\section{Neuro-modulatory treatments}

Unfortunately, there are no licensed drugs for the treatment of chronic cough, so in most specialist clinics management relies on using interventions with evidence of efficacy in clinical trials. The most commonly prescribed medication in our clinic is slow release morphine sulphate (MST) starting at $5 \mathrm{mg}$ twice daily and occasionally increasing to $10 \mathrm{mg}$ twice daily. ${ }^{39}$ If MST is ineffective then a discussion of the potential risks and benefits of trialling gabapentin or pregabalin is crucial. ${ }^{40,41}$ These drugs bind the $\alpha 2 \delta$ subunit of the voltage dependent calcium channel and are commonly used for neuropathic pain. The risks need to be carefully considered as unsteadiness, drowsiness, severe depression, hallucinations and, occasionally, suicidal ideation have been reported. Like MST, the benefits in cough have been reported by subjective improvements in the cough-specific quality of life questionnaire or a $100 \mathrm{~mm}$ visual analogue score. Low dose gapabentin has also been shown to be effective in cough syncope. ${ }^{42}$ Our practice with gabapentin has been to slowly up-titrate doses to $300 \mathrm{mg}$ three times a day and likewise with pregabalin, to a maximum of $150 \mathrm{mg}$ twice daily. This allows for an individualised dose for each patient and side effects are identified at lower doses. Amitriptyline $10 \mathrm{mg}$ at night has been assessed in a randomised controlled trial (RCT) without a placebo control, ${ }^{43}$ but our experiences with it have been disappointing.

Some patients decide against trying any of the above medication and opt for speech and language therapy (SALT). Given many patients report laryngeal symptoms and dysphonia, this can be an appealing option. One RCT and several observational studies have reported improvements in reported cough voice and quality of life. ${ }^{44}$ This intervention involves a multi-modality approach of education, reducing laryngeal irritation with relaxation exercises, cough suppression techniques and counselling. The major advantage is the lack of adverse events, but the disadvantage is that it is often dependent on the commitment of patients to continue their exercise routines outside the therapy sessions and on the expertise of the therapist in assessing and treating cough patients. Those that benefit often have SALT as an adjunct to medication and this was assessed in a 2016 RCT of SALT with placebo or pregabalin. ${ }^{41}$ The study showed that combining pregabalin with SALT improved cough-specific quality of life and visual analogue score, but disappointingly showed no additional improvement in actual objective cough frequency.

\section{Future directions}

There have been a number of studies using pharmacological therapies targeting specific receptors on nerve terminals either on the peripheral nerves or brainstem. Unfortunately, the vast majority have been negative, although one positive study targeting the $\mathrm{P} 2 \mathrm{X} 3$ receptor on peripheral nerves reported an unprecedented $75 \%$ reduction in 24 -hour objective cough frequency after just 2 weeks of treatment. ${ }^{45}$ This has prompted investigators in the field to further explore the role of ATP on nerves and cough, and it is hoped that results from a large multicentre study will pave the way for a future anti-tussive.

\section{Conclusion}

Despite the impression that chronic cough is a difficult condition to investigate and manage, a stepwise approach to investigate and identify associated treatable diseases can be successful. In the absence of treatment responses, neuromodulating pharmacological and physical therapies can provide relief, but this needs to be managed in specialist clinics. It is likely that over the coming years we will have novel anti-tussives targeting $\mathrm{P} 2 \mathrm{X} 3$ and other neuronal receptors with better efficacy and tolerability than current treatment options. 


\section{Conflicts of Interest}

AAW and JAS are named inventors on a patent, owned by University Hospital of South Manchester (UHSM), describing a method for generating output data licensed to Vitalograph Ltd; however, no financial benefits have been received

\section{References}

1 Cullinan P. Aetiological factors in persistent sputum production: a case-control study. J Epidemiol Community Health 1993;47:2731.

2 Ford AC, Forman D, Moayyedi P, Morice AH. Cough in the community: a cross sectional survey and the relationship to gastrointestinal symptoms. Thorax 2006;61:975-9.

3 French CL, Irwin RS, Curley FJ, Krikorian CJ. Impact of chronic cough on quality of life. Arch Intern Med 1998;158:1657-61.

4 Morice A, Kastelik JA, Thompson RH. Gender differences in airway behaviour. Thorax 2000;55:629.

5 Hilton E, Marsden P, Thurston A et al. Clinical features of the urge-to-cough in patients with chronic cough. Respir Med 2015;109:701-7.

6 McGarvey L, McKeagney P, Polley L, MacMahon J, Costello RW. Are there clinical features of a sensitized cough reflex? Pulm Pharmacol Ther 2009;22:59-64.

7 Smith SM, Schroeder K, Fahey T. Over-the-counter (OTC) medications for acute cough in children and adults in community settings. Cochrane Database Syst Rev 2014;(11):CD001831.

8 Canning BJ. Anatomy and neurophysiology of the cough reflex: ACCP evidence-based clinical practice guidelines. Chest 2006;129:33S-47S.

9 West PW, Canning BJ, Merlo-Pich E, Woodcock AA, Smith JA. Morphologic characterization of nerves in whole-mount airway biopsies. Am J Respir Crit Care Med 2015;192:30-9.

10 McGovern AE, Driessen AK, Simmons DG et al. Distinct brainstem and forebrain circuits receiving tracheal sensory neuron inputs revealed using a novel conditional anterograde transsynaptic viral tracing system. J Neurosci 2015;35:7041-55.

11 Young EC, Brammer C, Owen E et al. The effect of mindfulness meditation on cough reflex sensitivity. Thorax 2009;64:993-8.

12 Spring PJ, Kok C, Nicholson GA et al. Autosomal dominant hereditary sensory neuropathy with chronic cough and gastrooesophageal reflux: clinical features in two families linked to chromosome 3p22-p24. Brain 2005;128:2797-810.

13 Ford PA, Barnes PJ, Usmani OS. Chronic cough and Holmes-Adie syndrome. Lancet 2007;369:342.

14 Kimber J, Mitchell D, Mathias CJ. Chronic cough in the Holmes-Adie syndrome: association in five cases with autonomic dysfunction. Neurol Neurosurg Psychiatry 1998;65:583-6.

15 Baloh RH, Jen JC, Kim G, Baloh RW. Chronic cough due to Thr124Met mutation in the peripheral myelin protein zero (MPZ gene). Neurology 2004;62:1905-6.

16 Morice AH, Millqvist E, Belvisi MG et al. Cough hypersensitivity syndrome: clinical measurement is the key to progress. Eur Respir $J$ 2015;45:1509-10.

17 Hilton EC, Baverel PG, Woodcock A, Van Der Graaf PH, Smith JA. Pharmacodynamic modeling of cough responses to capsaicin inhalation calls into question the utility of the $\mathrm{C} 5$ end point. J Allergy Clin Immunol 2013;132:847-55 e1-5.

18 Sumner H, Woodcock A, Kolsum U et al. Predictors of objective cough frequency in chronic obstructive pulmonary disease. Am J Respir Crit Care Med 2013;187:943-9.

19 Morice AH, Kastelik JA. Cough. 1: Chronic cough in adults. Thorax 2003;58:901-7.
20 Irwin RS, Curley FJ, French CL. Chronic cough. The spectrum and frequency of causes, key components of the diagnostic evaluation, and outcome of specific therapy. Am Rev Respir Dis 1990;141:640-7.

21 Haque RA1, Usmani OS, Barnes PJ. Chronic idiopathic cough: a discrete clinical entity? Chest 2005;127:1710-3.

22 Belvisi MG, Birrell MA, Khalid S et al. Neuro-phenotypes in airway diseases: insights from translational cough studies. Am J Respir Crit Care Med 2016;193:1364-72.

23 Satia I, Tsamandouras N, Holt K et al. Capsaicin-evoked cough responses in asthmatic patients: Evidence for airway neuronal dysfunction. J Allergy Clin Immunol 2016; doi: 10.1016/j. jaci.2016.04.045. [Epub ahead of print]

24 Gibson P, Wang G, McGarvey L et al. Treatment of Unexplained Chronic Cough: CHEST Guideline and Expert Panel Report. Chest 2016;149:27-44.

25 Morice AH, Fontana GA, Sovijarvi AR et al. The diagnosis and management of chronic cough. Eur Respir J 2004;24:481-92.

26 Morice AH, Fontana GA, Belvisi MG et al. ERS guidelines on the assessment of cough. Eur Respir J 2007;29:1256-76.

27 Smith JA, Woodcock AA. Chronic Cough. N Engl J Med 2016;375:1544-51.

28 Yeo WW, Maclean D, Richardson PJ, Ramsay LE. Cough and enalapril: assessment by spontaneous reporting and visual analogue scale under double-blind conditions. Br J Clin Pharmacol 1991;31:356-9.

29 McFadden ER, Jr. Exertional dyspnea and cough as preludes to acute attacks of bronchial asthma. $N$ Engl J Med 1975;292:555-9.

30 Corrao WM, Braman SS, Irwin RS. Chronic cough as the sole presenting manifestation of bronchial asthma. $N$ Engl J Med 1979;300:633-7.

31 Brightling CE, Ward R, Goh KL, Wardlaw AJ, Pavord ID. Eosinophilic bronchitis is an important cause of chronic cough. Am J Respir Crit Care Med 1999;160:406-10.

32 Chang AB, Lasserson TJ, Gaffney J, Connor FL, Garske LA. Gastro-oesophageal reflux treatment for prolonged non-specific cough in children and adults. Cochrane Database Syst Rev 2011;(1):CD004823.

33 Kahrilas PJ, Howden CW, Hughes N, Molloy-Bland M. Response of chronic cough to acid-suppressive therapy in patients with gastroesophageal reflux disease. Chest 2013;143:605-12.

34 Badri H, Satia I, Woodcock AA, Smith JA. The usefulness of heartburn as a marker of the success of acid suppression therapy in chronic cough [abstract]. Am J Respir Crit Care Med 2015;191:A4124.

35 Grabowski M, Kasran A, Seys S et al. Pepsin and bile acids in induced sputum of chronic cough patients. Respir Med 2011;105:1257-61.

36 Pauwels A, Decraene A, Blondeau K et al. Bile acids in sputum and increased airway inflammation in patients with cystic fibrosis. Chest 2012;141:1568-74.

37 Decalmer S, Stovold R, Houghton LA et al. Chronic cough: relationship between microaspiration, gastroesophageal reflux, and cough frequency. Chest 2012;142:958-64.

38 Birring SS, Passant C, Patel RB et al. Chronic tonsillar enlargement and cough: preliminary evidence of a novel and treatable cause of chronic cough. Eur Respir J 2004;23:199-201.

39 Morice AH, Menon MS, Mulrennan SA et al. Opiate therapy in chronic cough. Am J Respir Crit Care Med 2007;175:312-5.

40 Ryan NM, Birring SS, Gibson PG. Gabapentin for refractory chronic cough: a randomised, double-blind, placebo-controlled trial. Lancet 2012;380:1583-9.

41 Vertigan AE, Kapela SL, Ryan NM et al. Pregabalin and speech pathology combination therapy for refractory chronic cough: a randomized controlled trial. Chest 2016;149:639-48. 
$42 \mathrm{Wu} \mathrm{CH}$, Gunasekara K, Hull JH et al. Gabapentin as a potential treatment for cough syncope. Respir Care 2015 Feb;60:e34-7.

43 Jeyakumar A, Brickman TM, Haben M. Effectiveness of amitriptyline versus cough suppressants in the treatment of chronic cough resulting from postviral vagal neuropathy. Laryngoscope 2006;116:2108-12.

44 Vertigan AE, Theodoros DG, Gibson PG, Winkworth AL. Efficacy of speech pathology management for chronic cough: a randomised placebo controlled trial of treatment efficacy. Thorax 2006;61:1065-9.
45 Abdulqawi R, Dockry R, Holt K et al. P2X3 receptor antagonist (AF-219) in refractory chronic cough: a randomised, double-blind, placebo-controlled phase 2 study. Lancet 2015;385:1198-205.

Address for correspondence: Prof A Woodcock, Division of Infection, Immunity and Respiratory Medicine, University of Manchester. Level 2, Education and Research Centre, University Hospital of South Manchester, Southmoor Road, Manchester M23 9LT, UK.

Email: ashley.woodcock@manchester.ac.uk 